\title{
Lower Bound on the Propagation Speed of Gravity from Gravitational Cherenkov Radiation
}

\author{
Guy D. Moore and Ann E. Nelson \\ Department of Physics, University of Washington \\ Seattle, Washington, WA 98195, USA.
}

\begin{abstract}
Recently, interesting 4-D Lorentz violating models have been proposed, in which all particles have a common maximum velocity $c$, but gravity propagates (in the preferred frame) with a different maximum velocity $c_{g} \neq c$. We show that the case $c_{g}<c$ is very tightly constrained by the observation of the highest energy cosmic rays. Assuming a galactic origin for the cosmic rays gives a conservative bound of $c-c_{g}<2 \times 10^{-15} c$; if the cosmic rays have an extragalactic origin the bound is orders of magnitude tighter, of order $c-c_{g}<2 \times 10^{-19} c$.
\end{abstract}

KEYwords: Asymmetrical warping, extra dimensions, Cherenkov radiation, Lorentz violation. 


\section{Introduction and Motivation}

A new generation of experiments utilizing long baseline interferometers is commencing to search for gravitational waves [1, 2, 3, 4, $4 .{ }^{1}$ Within ten years or so it is quite possible that gravitational waves from astrophysical sources will have been observed. As well as opening a new astronomical and cosmological window, this may allow for tests of unprecedented accuracy of the general relativistic prediction for the speed and polarization of gravitational waves. Accurate measurement of the speed of propagation of gravitational waves can constrain extra-dimensional "brane-world" theories in which gravity propagates in the bulk of extra dimensions [8, 9, 10, 11, 12, 13, 14, while the particles of the Standard Model are confined to a $3+1$ dimensional subspace known as a brane. It has been argued that in many cases Poincaré invariance should be violated in the bulk [15, 16, 17, 18, 19, 20, 21] giving rise to an anomalous dispersion relation for gravitational waves. ${ }^{2}$ No Lorentz violation would show up in the standard model, provided our brane is Poincaré invariant.

The ADS/CFT correspondence [22] has given rise to a purely 4 dimensional interpretation, in the infrared, of warped higher dimensional geometry 223, 24, 25, 26, 27, 18]. It is conjectured that a bulk with 4-dimensional Poincaré invariance and 5-dimensional antideSitter (AdS) invariance may be given a dual 4-dimensional interpretation as a dark 'conformal sector' which interacts gravitationally. A less symmetric bulk may be regarded simply as deviations of the dark sector from conformality and/or Poincaré invariance. Deviations from Poincaré invariance would be expected if the conformal sector were at finite temperature. If gravitational deviations from Poincaré invariance can be interpreted simply as a modified dispersion relation for gravitational waves due to interaction with a hot dark sector, then gravitational waves always travel slower than light [28]. ${ }^{3}$

In this note we remark that if gravity is slower than light, one expects particles moving faster than the speed of gravity to emit "gravi-Cherenkov radiation," in analogy with the Cherenkov radiation emitted by particles moving faster than light in a medium. The existence of high energy cosmic rays which have travelled from astronomical distances without losing

\footnotetext{
${ }^{1}$ for reviews, see ref. [5, 6, , 7].

${ }^{2}$ In ref. 17] it was argued on the basis of examining geodesics that in such Poincaré non-invariant warped geometries gravity would always travel at least as fast as light. The argument is that if the bulk velocity were slower than light, gravity could just stay on the brane. Such a geodesic analysis actually establishes the maximum propagation speed over all Kaluza-Klein states of the graviton with any wave number, rather than the propagation speed of the massless graviton. The constraint we find in this paper applies if the graviton or any KK state propagates with subluminal phase velocity when its energy is less than that of the most energetic cosmic rays.

${ }^{3}$ In this case, if we enforce the additional constraint that the energy density off the brane is less than the critical density $\rho_{\text {crit }}=8 \pi G_{\mathrm{N}} H_{0}^{2} / 3$, as seems necessary on cosmological grounds, then the propagation velocity is strongly constrained. On wavelengths longer than the compactification radius, the gravitational waves will have the dispersion relations of 4-D gravitational waves interacting with (off-brane) dark matter. If the dark matter behaves as dust, the frequency $\omega$ dependent propagation speed is $\left(c-c_{g}\right) / c \simeq 2 \pi G \rho / \omega^{2}$ [28]; for other equations of state the coefficient will differ but the form should be the same. If the compactification radius is, say, $1 \mathrm{~mm}$, the velocity difference for wavelength of order the compactification radius is $\left(c-c_{g}\right) / c<10^{-58}$.
} 
all their energy to gravi-Cherenkov radiation places a strong lower bound on the speed of gravitational waves with very short wave lengths (energies of order $10^{10} \mathrm{GeV}$ ).

We begin by reviewing Cherenkov radiation from a particle physics point of view. Then we generalize to gravitational Cherenkov radiation, and apply it to bound the speed of propagation of gravitational waves. Similar limits have been placed on Lorentz violating effects in which different standard model particles have different maximum propagation velocities, by considering ordinary Cherenkov radiation [29, 30]. Our constraints will turn out to be weaker because the efficiency of gravitational Cherenkov radiation is orders of magnitude less than the efficiency of ordinary Cherenkov radiation.

\section{Cherenkov radiation}

Cherenkov radiation occurs when a charged particle moves faster than the local (in-medium) speed of light. Intuitively, Cherenkov radiation happens because the charged particle "outruns" its own electromagnetic field. It is easiest to see that this leads to energy loss by considering the case where the speed of electromagnetic propagation is zero; then the electric flux of a charge will trail out along its past trajectory. As the charge moves, it lengthens the trajectory and pays the energy cost of the added electric field energy.

In normal applications Cherenkov radiation occurs when some medium effect causes the propagation speed of electromagnetic radiation to be less than light speed, for light with frequencies below some cutoff frequency which is typically set by an atomic excitation frequency. In this case the index of refraction varies with frequency in an important way, and Cherenkov radiation only occurs for frequencies much less than the energy of the relativistic charged particle. Therefore a classical treatment is applicable. For simplicity, however, we will consider cases where the index of refraction $n$ is frequency independent in the deep ultraviolet. We also only need treat cases where $c_{g}$ is very close to the maximum propagation speed of the particle, that is, the index of refraction $n=c_{\text {particles }} / c_{\text {light }}=1+\epsilon$ with $\epsilon \ll 1$ a constant. Cherenkov radiation corresponds to the process shown in Fig. 1. Normally this process

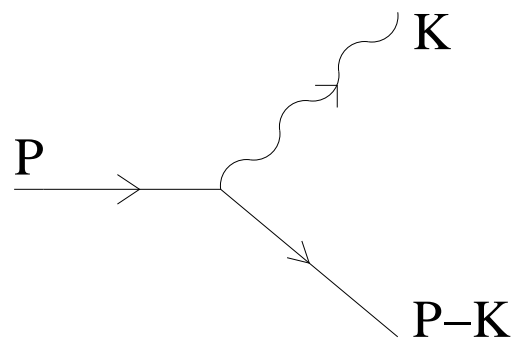

Figure 1: Diagram responsible for Cherenkov radiation.

is kinematically forbidden and we would never consider it. However, the dispersion relation for a photon with a constant index of refraction $n>1$ is $|\mathbf{k}|=n k_{0}$. Therefore, for $n>1$ a photon carries more momentum than energy, which makes the process kinematically allowed 
for sufficiently relativistic charge carriers. Note that it is the phase velocity of the light which is important here, not the group velocity.

Evaluating the two-body decay rate by standard techniques, and approximating $n \simeq 1$ whenever it is safe to do so, gives a photon emission rate from a scalar of charge $e$, mass $m$ and momentum $\mathbf{p}$ of

$\frac{d n_{\gamma}}{d t}=\frac{1}{2 p_{0}} \int \frac{d^{3} \mathbf{k} d k_{0}}{(2 \pi)^{4}} 2 \pi \delta\left[n^{2} k_{0}^{2}-\mathbf{k}^{2}\right] 2 \pi \delta\left[\left(p_{0}-k_{0}\right)^{2}-(\mathbf{p}-\mathbf{k})^{2}-m^{2}\right] e^{2} \sum_{\epsilon}\left((2 P-K)^{\mu} \epsilon_{\mu}\right)^{2}$

where the sum is over the two transverse photon polarization states $\epsilon$. The first delta function forces the photon to be on the (modified) mass shell $|\mathbf{k}|=n k_{0}$ and the second is the mass shell condition for the outgoing scalar, while the final factor is the square of the matrix element. Writing $|\mathbf{p}| \equiv p$ and $|\mathbf{k}| \equiv k$, and defining $\theta$ to be the angle between $\mathbf{p}$ and $\mathbf{k}, \mathbf{p} \cdot \mathbf{k}=p k \cos \theta$, the second delta function can be rewritten as

$$
\begin{aligned}
\delta\left[\left(p_{0}-k_{0}\right)^{2}-(\mathbf{p}-\mathbf{k})^{2}-m^{2}\right] & =\delta\left[2 p k \cos \theta-2 p_{0} k_{0}-k^{2}+k_{0}^{2}\right] \\
& =\frac{1}{2 p k} \delta\left[\cos \theta-\frac{1}{\beta n}-\frac{\left(n^{2}-1\right) k}{2 n^{2} p}\right]
\end{aligned}
$$

where we have written the propagation velocity of the incoming charged particle as $p / p_{0} \equiv$ $\beta \simeq 1$. The photon emission rate becomes

$$
\frac{d n_{\gamma}}{d t}=\frac{e^{2}}{4 \pi} \int_{0}^{k_{\max }} d k \sin ^{2} \theta, \quad \cos \theta=\frac{1}{\beta n}+\frac{\left(n^{2}-1\right) k}{2 n^{2} p}, \quad k_{\max }=p \frac{(\beta n)-1}{\beta(n-1)},
$$

where, note, we have already approximated $(n-1) \ll 1$ and $(1-\beta) \ll 1$. The small $k$ limit is the same as the classical electromagnetic result. The power emitted is obtained by inserting a factor of $k$ in the integrand. Note that, for $k \sim p$, spin dependent corrections become $O(1)$.

\section{Gravitational Cherenkov radiation}

The rate of energy loss by gravitational Cherenkov radiation is very similar. There are a few key physical differences, however. Rather than the electric charge $e^{2}$, it is the gravitational constant times the energy squared, $G_{\mathrm{N}} p^{2}$, which will appear as the prefactor. Also, the tensor nature of the gravitational interaction reduces emission at small opening angle, so it is $\sin ^{4} \theta$, rather than $\sin ^{2} \theta$, which appears. The same diagram, Fig. 1, is responsible for gravitational Cherenkov radiation, with the wavy line now representing a graviton rather than a photon. The relevant Feynman rules can be obtained from Appendix A of [31]. ${ }^{4}$ The rate of graviton

\footnotetext{
${ }^{4}$ The Feynman rules in [31] are for arbitrary KK states of the graviton and must be truncated for our purposes; only the spin \pm 2 polarization states in Eq. (A.3) of that paper are to be summed over, and indices referring to KK state number are to be ignored.
} 
emission by gravitational Cherenkov radiation, again taking $(n-1) \ll 1$ and $(1-\beta) \ll 1$, is

$$
\begin{gathered}
\frac{d n_{\text {grav }}}{d t}=\frac{1}{2 p} \int \frac{d^{3} \mathbf{k} d k_{0}}{(2 \pi)^{4}} 2 \pi \delta\left[n^{2} k_{0}^{2}-\mathbf{k}^{2}\right] 2 \pi \delta\left[\left(p_{0}-k_{0}\right)^{2}-(\mathbf{p}-\mathbf{k})^{2}-m^{2}\right] \times \\
\times 16 \pi G_{\mathrm{N}} \sum_{\epsilon}\left(\epsilon^{\mu \nu} P_{\mu}(P-K)_{\nu}\right)^{2}
\end{gathered}
$$

where the sum is over the two transverse traceless graviton polarizations, which project out the components of $P_{\mu},(P-K)_{\mu}$ orthogonal to $K_{\mu}$. The result for energy loss per unit time is obtained by multiplying the integrand by $k$, and is

$$
\frac{d E}{d t} \simeq G_{\mathrm{N}} p^{2} \int_{0}^{k_{\max }} k d k \sin ^{4} \theta
$$

where $k_{\max }$ and $\theta$ are the same as in Eq. (2.3). If we take the limit $(1-\beta) \ll(n-1)$, which will be appropriate to our application, then the simpler expressions $k_{\max } \simeq p$ and $\sin ^{2} \theta \simeq$ $\theta^{2} \simeq 2(n-1)(1-k / p)$ hold, and the integral is easy $;^{5}$

$$
\frac{d E}{d t} \simeq 4 G_{\mathrm{N}}(n-1)^{2} \int_{0}^{p}(p-k)^{2} k d k=\frac{G_{\mathrm{N}} p^{4}(n-1)^{2}}{3} .
$$

The energy loss arises predominantly by shedding gravitons with energy $k \lesssim p / 2$. We have presented this calculation for scalars, but we have checked that the result is the same for fermions.

Integrating this equation, the relation between the travel time, the initial momentum $p_{\text {init }}$, and the final momentum $p$ is

$$
t_{\text {travel }}=\frac{1}{G_{\mathrm{N}}(n-1)^{2}}\left(\frac{1}{p^{3}}-\frac{1}{p_{\text {init }}^{3}}\right) .
$$

Therefore, a particle of momentum $p$ cannot possibly have been traveling for longer than $t_{\max }=m_{\mathrm{pl}}^{2} /(n-1)^{2} p^{3}$.

\section{Application to cosmic rays}

Several cosmic rays have been observed with energies in excess of $10^{11} \mathrm{GeV} 33$. The highest energy cosmic ray which has been observed was probably a proton, of energy $\sim 3 \times 10^{11} \mathrm{GeV}$ [34]. A proton is a composite object. Viewed at the energy scale $k \sin \theta \sim 10^{3} \mathrm{GeV}$, it is made up of pointlike partons with typical momentum fraction $x \sim 1 / 10$. Let us assume conservatively that the proton arrived at Earth from a distance of order the distance to

\footnotetext{
${ }^{5}$ A result similar to Eq. 3.3 has been derived by Pardy [32], for general $\beta<1$ and $n>1$ but using a classical technique only applicable for $k \ll p$. After correcting an error in Eq. (19) there [his $\left(n^{2} / \beta^{2}+1\right)^{2}$ should read $\left.\left(n^{2} / \beta^{2}-1\right)^{2}\right]$ his answer agrees with ours in the overlapping domain of validity, $k \ll p$ and $(1-\beta) \ll(n-1) \ll 1$.
} 
the galactic center, say $\sim 10 \mathrm{kpc}$ (Kiloparsec). In particle physics units, $10 \mathrm{kpc} \simeq 1.57 \times$ $10^{36} \mathrm{GeV}^{-1}$. Using the bound on the time of flight, Eq. (3.4), we find

$$
n-1 \leq \sqrt{\frac{m_{\mathrm{pl}}^{2}}{(0.1 E)^{3} t}} \simeq 2 \times 10^{-15} .
$$

Such an $n$ indeed satisfies $(1-\beta) \simeq m^{2} / 2 E^{2} \sim 10^{-23} \ll(n-1) \ll 1$, so our analysis is self-consistent. This bound is strong enough that coincidence tests between gravitational wave detectors and other observations (gamma rays, etc.) of distant gravity wave sources are unlikely to do better.

Our result is also very conservative. For instance, we neglect that the emission of a graviton with transverse momentum $k \sin \theta \sim 100 \mathrm{GeV}$ would break up the proton, so the actual energy loss to the proton would be substantially more than the energy of the graviton. Also, in many higher dimensional theories there will be KK states of the graviton with mass $\lesssim 100 \mathrm{GeV}$. These will also satisfy $|\mathbf{k}|>k_{0}$ at energies $k_{0} \sim 10^{10} \mathrm{GeV}$, so the proton will radiate them as well, increasing the efficiency of the energy loss mechanism. For instance, if there is one extra millimeter scale dimension, our bound strengthens by $\sim 7$ orders of magnitude.

Our bound also assumes that there be some exotic physics which can generate the highest energy cosmic rays within the galactic halo. The result tightens if we do not assume such exotic physics. This is an experimental question which will be resolved when the angular distribution on the sky of the highest energy cosmic rays is determined [35]. Consider instead the possibility that the highest energy cosmic rays are produced by the "Z-burst" mechanism, in which very high energy neutrinos produced at cosmological distances annihilate with relic neutrinos via the $Z$ boson resonance [36, 37]. In this case, the energy loss of the high energy neutrino sets a limit on $n-1$ which is much tighter. The energy of the neutrino must exceed the energy of the cosmic ray primary, $\sim 3 \times 10^{11} \mathrm{GeV}$, after traveling a cosmological distance, say $\sim 2 \mathrm{Gpc} \sim 3 \times 10^{41} \mathrm{GeV}^{-1}$. In this case the bound becomes

$$
n-1 \leq 1.3 \times 10^{-19}
$$

This bound is much tighter but is model dependent.

We can say less about the case $n<1$, where gravity propagates faster than the speed of light. In this case gravitational Cherenkov radiation is impossible. On the other hand, in terms of the particle physics Lorentz metric, a graviton has a timelike energy 4 -vector, so it is kinematically allowed for a graviton to convert into, say, two photons. However, as we have seen, the reaction rate is very highly energy dependent, so the relatively low frequency gravity waves we expect from most gravity wave producing phenomena are completely unaffected. Therefore the direct observational bounds on $n<1$ are set by precision tests of gravity, and are very much weaker than for $n>1$. 


\section{References}

[1] A. Abramovici et. al., Ligo: The laser interferometer gravitational wave observatory, Science 256 (1992) 325-333.

[2] A. Giazotto, The virgo project: A wide band antenna for gravitational wave detection, Nucl. Instrum. Meth. A289 (1990) 518-525.

[3] B. F. Schutz, Getting ready for GEO600 data, Prog. Theor. Phys. Suppl. 136 (1999) 168, gr-qc/9910033.

[4] TAMA Collaboration, H. Tagoshi et. al., The first search for gravitational waves from inspiraling compact binaries using TAMA300 data, Phys. Rev. D63 (2001) 062001, gr-qc/0012010.

[5] K. S. Thorne, Gravitational waves, gr-qc/9506086.

[6] K. S. Thorne, Gravitational radiation: A new window onto the universe, gr-qc/9704042.

[7] C. M. Will, The confrontation between general relativity and experiment, Living Rev. Rel. 4 (2001) 1 , gr-qc/0103036.

[8] K. Akama, An early proposal of 'brane world', Lect. Notes Phys. 176 (1982) 267-271, hep-th/0001113.

[9] B. Holdom, The cosmological constant and the embedded universe, . ITP-744-STANFORD.

[10] V. A. Rubakov and M. E. Shaposhnikov, Do we live in a domain wall?, Phys. Lett. 125B (1983) 136.

[11] N. Arkani-Hamed, S. Dimopoulos, and G. Dvali, The hierarchy problem and new dimensions at a millimeter, Phys. Lett. B429 (1998) 263, hep-ph/9803315.

[12] I. Antoniadis, N. Arkani-Hamed, S. Dimopoulos, and G. Dvali, New dimensions at a millimeter to a fermi and superstrings at a TeV, Phys. Lett. B436 (1998) 257, hep-ph/9804398.

[13] L. Randall and R. Sundrum, A large mass hierarchy from a small extra dimension, hep-ph/9905221.

[14] L. Randall and R. Sundrum, An alternative to compactification, hep-th/9906064.

[15] D. J. H. Chung and K. Freese, Cosmological challenges in theories with extra dimensions and remarks on the horizon problem, Phys. Rev. D61 (2000) 023511, hep-ph/9906542].

[16] H. Ishihara, Causality of the brane universe, Phys. Rev. Lett. 86 (2001) 381-384, gr-qc/0007070.

[17] C. Csaki, J. Erlich, and C. Grojean, Gravitational lorentz violations and adjustment of the cosmological constant in asymmetrically warped spacetimes, hep-th/0012143.

[18] A. Hebecker and J. March-Russell, Randall-Sundrum II cosmology, AdS/CFT, and the bulk black hole, hep-ph/0103214.

[19] J. W. Moffat, Acceleration of the universe, string theory and a varying speed of light, hep-th/0105017.

[20] D. Youm, Variable-speed-of-light cosmology from brane world scenario, hep-th/0102194. 
[21] R. R. Caldwell and D. Langlois, Shortcuts in the fifth dimension, gr-qc/0103070.

[22] J. Maldacena, The large $N$ limit of superconformal field theories and supergravity, Adv. Theor. Math. Phys. 2 (1998) 231-252, hep-th/9711200.

[23] H. Verlinde, Holography and compactification, Nucl. Phys. B580 (2000) 264-274, hep-th/9906182.

[24] E. Witten, Remarks at ITP Santa Barbara conference on 'new dimensions in field theory', http://www.itp.ucsb.edu/online/susy_c99/discussion/,

[25] S. S. Gubser, AdS/CFT and gravity, Phys. Rev. D63 (2001) 084017, hep-th/9912001.

[26] N. Arkani-Hamed, M. Porrati, and L. Randall, Holography and phenomenology, hep-th/0012148.

[27] R. Rattazzi and A. Zaffaroni, Comments on the holographic picture of the Randall-Sundrum model, JHEP 04 (2001) 021, hep-th/0012248.

[28] P. C. Peters, Index of refraction for scalar, electromagnetic, and gravitational waves in weak gravitational fields, Phys. Rev. D9 (1974) 2207-2218.

[29] S. Coleman and S. L. Glashow, High-energy tests of Lorentz invariance, Phys. Rev. D59 (1999) 116008, hep-ph/9812418.

[30] F. W. Stecker and S. L. Glashow, New tests of Lorentz invariance following from observations of the highest energy cosmic gamma rays, astro-ph/0102226.

[31] T. Han, J. D. Lykken, and R.-J. Zhang, On Kaluza-Klein states from large extra dimensions, Phys. Rev. D59 (1999) 105006, hep-ph/9811350.

[32] M. Pardy, The gravitational cerenkov radiation with radiative corrections, Phys. Lett. B336 (1994) 362-367.

[33] M. Nagano and A. A. Watson, Observations and implications of the ultrahigh-energy cosmic rays, Rev. Mod. Phys. 72 (2000) 689-732.

[34] D. J. Bird et. al., Detection of a cosmic ray with measured energy well beyond the expected spectral cutoff due to comic microwave radiation, Astrophys. J. 441 (1995) 144-150.

[35] S. L. Dubovsky and P. G. Tinyakov, Galactic anisotropy of ultra-high energy cosmic rays produced by CDM-related mechanisms, hep-ph/9810401.

[36] T. Weiler, Resonant absorption of cosmic ray neutrinos by the relic neutrino background, Phys. Rev. Lett. 49 (1982) 234.

[37] T. J. Weiler, Cosmic ray neutrino annihilation on relic neutrinos revisited: A mechanism for generating air showers above the Greisen-Zatsepin-Kuzmin cut-off, Astropart. Phys. 11 (1999) 303-316, hep-ph/9710431. 